International Journal of Distributed and Parallel Systems (IJDPS) Vol.1, No.2, November 2010

\title{
FORWARDING OF MULTIPLE MESSAGES TO MULTIPLE DESTINATIONS BY USING MOBILE AGENTS
}

\author{
G.V. Shivani ${ }^{1}$ and M.Dinakaran ${ }^{2}$ \\ ${ }^{1}$ School of IT \& Engineering, VIT University, Vellore, South India. \\ gv.shivani1@gmail.com \\ ${ }^{2}$ School of IT \& Engineering, VIT University, Vellore, South India. \\ dinakaran.m@vit.ac.in
}

\begin{abstract}
Today in this competitive world where everybody depends on the mobile technology run with the need of possessing mobiles and enjoying the facilities of ease to send messages to their near and dear ones. People need to perform their tasks for informing, contacting and keeping an up to date of whatever is going around them. One such facility in our mobiles is forwarding of messages to people for fun or for awareness or when we want more number of people to know about something. The proposal is forwarding multiple messages to multiple destinations at the same time. The mobiles have the facility to forward a single message to multiple destinations. The messages can be forwarded using the push approach and the Mobile Agent Client and Mobile Agent Server. Since a mobile agent has certain properties which supports multiple message forwarding but affects the reliability certainly, mobile agents for sub servers can be a substitute as well. Messages will be selected in an order and the recipients' numbers are be added. Once the sender toggles the option of 'send', the multiple messages selected must be sent to the recipients in the order they are selected to send. It can either be based on the messages like one by one message must first be sent to the recipients thus all the messages selected reaches the recipient 1 then all the messages will be sent to the next recipient 2 and so on or message 1 is sent to all the recipients and message 2 is sent to all the recipients and so on. Gateways in Mobile Agent Server can be used for the store and forward technique. If the network is busy and any message is not received by any of the recipient then the Mobile Agent Server will locate the address from the MA Client and establish connectivity again and then forwards the message.
\end{abstract}

\section{KEYWORDS}

Mobile Agent Client, Mobile Agent Server, Forward multiple messages, Gateway (message hub)

\section{INTRODUCTION}

Mobile Agents are autonomous objects which enable movement between locations in the MA system. An MA system is a distributed abstraction layer that provides mobility and communication as well as security. MAs also provide a convenient and powerful paradigm for structuring distributed systems and applications. Agents can travel over the network to search for, filter, and process information required to accomplish their tasks. They can also cooperate with each other by sharing and exchanging information and partial results, and collectively making decisions. In various situations, MAs need to communicate with each other by passing messages. Remote inter agent communication is thus a fundamental facility in MA systems. Mobile Agents generally involve the relay communication style in which multiple senders will 
be present at one MAP and multiple recipients will be present at another MAP. MAP is MA platform (Mobile Agent Platform) which provides communication facility, security and mobility support for MA's [1][5].

Mobile Agents have a great potential in the distributed network architecture as they provide structuring of the distributed systems and applications. These Agents actually enable sharing of information, cooperative work between MA systems, search and filter selective information, share work and partial results and gather information required to perform their tasks completely. Mobility of the Agents needs to be considered for mainly two reasons a) location of MA has to be found b) message delivery to the agent. In few MA systems, certain proxies are used to implement the message forwarding by tracking the agents, location transparency. Proxy will hold all the messages sent to the MA and from there a current location address will be handled and to that current address messages are forwarded [1][5].

Mobile Agents are software located in the network which based on movement dynamically searches for the required destinations and if not present in the network will add the destination address virtually and perform data transfer. Protocols based on the relay communication model can effectively track the location of MAs and implement location transparent communication, but additional efforts are needed to guarantee reliable delivery of messages to MAs. Here, by reliability we mean that no matter how frequently the target agent migrates, messages will be routed to it in a bounded number of hops without being dropped. Even with an ideal fault-free network transport mechanism, messages are not guaranteed to be reliably delivered to their destination MAs. Since there are disadvantages because of this as well, the method of push and pull is used [1].

There are two approaches which are going to be discussed namely push and pull in Mobile Agents. In the push mode, the messages are forwarded one by one when they are encountered in an MA. In pull mode, the messages delivered have an address and based on that address request is processed at some interval of time. Two types of pull operation are already known Greedy pull and Distance based pull for reducing the message delay and network traffic.

In the following proposal we are using the concept of Mobile Agent Client and Mobile Agent Server. In a Mobile Agent Platform (MAP) all the senders and recipients will be involved and the Mobile Agent Server will perform connectivity with the Mobile Agent Client using a concept of Gateway to enhance the performance of forwarding multiple messages to multiple destinations [1][2].

Here we will discuss how the Mobile Agent Client will use the push and pull operation in order to form connectivity with the Mobile Agent Server. Mobile Agent Server will hold all the details of Clients and proceed to forwarding of messages [1][2][5].

\section{MOBILE AGENT CLIENT}

MA Client is the Mobile Agent Client which is going to perform all the client side activities that are required under one particular network and secondly it will establish connectivity with the other networks as well in order to perform the message forwarding reliably. MA Client is going to work on the push mode used for Mobile Agents in order to increase the performance by enhancing the relay type of communication in MAP and reduce the message delay. 
In the Mobile Agent Client, push mode is going to forward each message as one by one comes into the network visibility. This push operation will be performed by MA Client which holds multiple senders and recipients in the network and sender's process of searching for the recipients and forwarding the packets is detected by MA Server. Each sender can be an enabled node in the network throughout the time period in which they will send the messages or vice versa. Using the push mode, a particular location will be saved or allotted to a particular receiver. Receivers are the target agents and the messages are going to be allotted one by one based on the location and the location information is retrieved from the database. Since the receivers are mobile in nature, there is a complete chance of message delivery failure and then we cannot guarantee reliable message delivery. Hence Synchronization is very important. Synchronizing the senders with receivers is achieved by updating the location of the receivers continuously so that the flow of messages is not lost. The address which is stored based on the location needs to be updated simultaneously in order to track another receiver which leaves that particular location of which the detail was stored. Synchronization is important as there are chances of failure of complete delivery of message because of the mobility of the systems $[1][5][6]$.

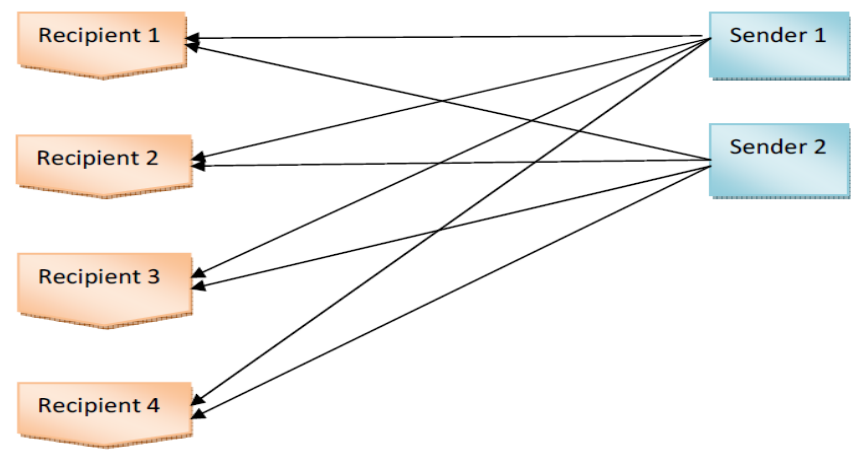

Fig 1: Multiple recipients will receive messages from multiple senders

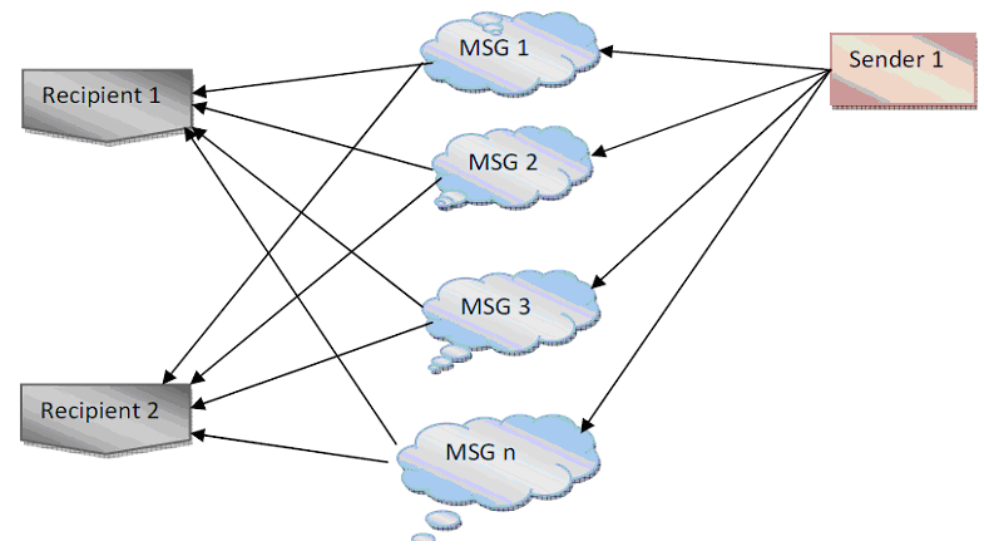

Fig 2: Multiple messages being sent by the Sender to multiple recipients. 


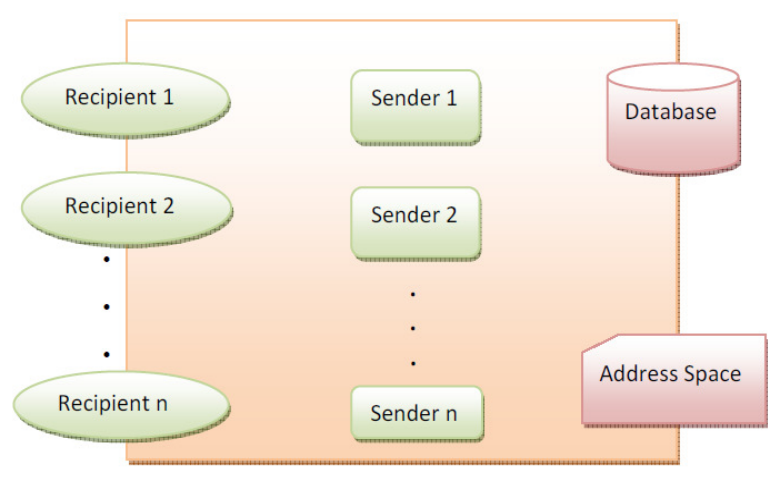

Fig 3: Represents the Objects on the Mobile Agent Client side

Above are the objects in a Mobile Agent Client multiple senders, multiple receivers, database, address space. A sender will select multiple messages and multiple recipients, and toggles the forward option. Since the address space carries all the contacts or addresses in the senders mobile, there is less chance of message failure since the node to be absent from the network will not happen. The database will try to get connectivity from Mobile Agent Server to perform the request toggled by the senders [1][2].

\subsection{Properties of Push Mode in MA Client}

There are few properties of this push approach as it is mainly related to Mobile Agents. Following are few of them:

- Reliability: Reliability actually means delivery of message must be done. For the proper delivery of message, synchronization within the network area or number of hops present is very important.

- Resiliency to Failures: There must be recovery for every message that fails to be delivered. After being recovered from the fail state it must be forwarded to the destined MA system or agent.

- Support of Real Time Message Processing: In this push mode, the messages need to be forwarded as soon as they arrive at the relay station. Here the acknowledgement is very important. In the pull mode, relay station is queried every now and then due to which the delay time needs to be checked in addition to the transmission mode.

- Flexibility: Since the agent has the autonomy to decide on the time and frequency of the queries for messages, more flexibility is introduced in the pull mode. For example, the agent can adjust its query frequency dynamically. If it is in urgent need of information from its coordinator, it may query at a higher frequency. Otherwise, a lower frequency is adopted. Distance can be another factor of concern. If the current location of the agent is very far from its relay station, it can query the relay station at a much lower frequency or does not query at all. When it migrates to a host nearer to its relay station, it can query more frequently and process more messages buffered in the relay station [1][5]. 


\section{MOBILE AGENT SERVER}

Mobile Agent Server plays a very crucial role because the whole of the connectivity depends upon the MA Server. Here Gateways are used for proper connectivity issues. Gateways are message hubs which facilitate the store and forward technique and provide reliable transfer of messages as well as optimization. MA Server will establish a connection with the MA Client and gets all the messages that are selected to be forwarded and stores a copy of it in it database. The buffer space which holds the messages in the database can be updated each time the senders need to forward messages to the recipients. In a network as known there are loads of senders and receivers. Hence to save storage space and use it efficiently and optimize it, buffers can be regularly updated. As soon as the buffer receives messages to be forwarded, it will create a few number of copies of it based on the selection of the sender (how many number of recipients one needs to forward the message). Based on that number, that many copies of the messages are created and forwarded [2][5][9].

If the Server fails just in case then Mobile Agents can communicate using the sub servers. Sub Servers will act as the main Server and forward the messages. They take the complete charge when the main Server is down. Once the main Server resumes, it starts its work again. When the main Server breaks down, a request is sent to it. If it does not respond to that request, then it is considered to be down. Then the sub server will perform the operation in order to reduce the delay [10][11][12][13].

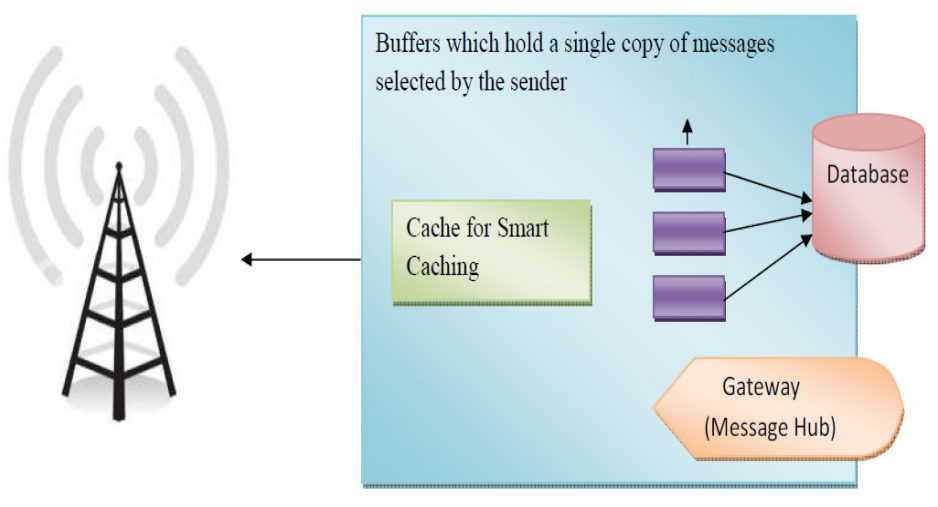

Fig 4: Mobile Agent Server side objects and functionalities.

The Base Station checks for signal presence and accordingly connectivity is established. The Gateway has a very important feature that is the SmartCaching. SmartCaching is basically involved in Gateways because the multiple messages need to be stored and forwarded. And the buffer has to be maintained. Since usage of space happens on a regular basis, caching is very important. Caching is essential for improving the performance by reducing network traffic and latency. Sometimes same message can be forwarded again and again hence this cache will store some messages and reuse it and then when the validity of that message is over in the cache it will be removed [6][7][13]. 


\section{OVERALL ARCHITECTURE}

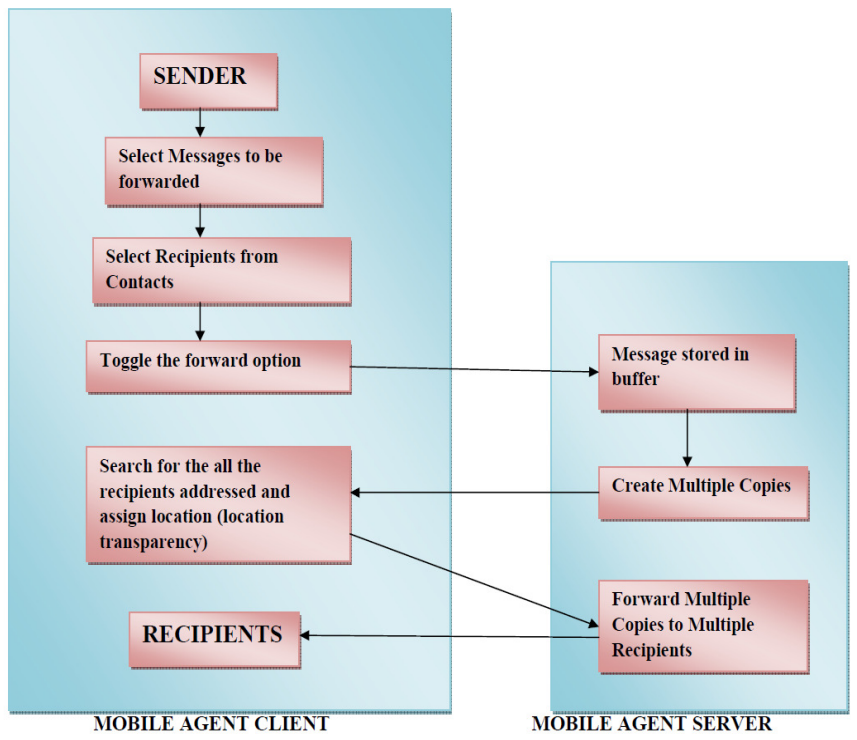

Fig 5: Detailed Explanation of the proposed architecture

In the above architecture, the operations taken care by Mobile Agent Client and Mobile Agent Server are denoted clearly.

The Mobile Agent Client side the senders and the receivers reside. As in mobile phones, the senders will select all the inbox messages required and add the contact numbers. Once they toggle the "forward" button, the messages selected and the recipient details must be sent to the Mobile Agent Server which will create multiple replicas of the messages and based on the recipients information, the messages will be buffered and the recipients are searched and the multiple copies of messages are sent.

The base station which is located will help contact the Service Providers which actually forwards the messages and recipient information to the Mobile Agent which is a software located in the network and will route and forward the messages based on location transparency. Thus the communication between mobile devices takes place.

The Mobile Agent Server will receive the request from the Mobile Agent Client and based on the number of recipients it will create those many replicas of the messages received. The buffer will contain the messages to be sent and updated each time. The SmartCaching technique is the property of the gateways which is a message hub and helps perform connectivity with the Clients. This cache contains all the messages which haven't been sent to the recipients due to network connection failure. Once the connection is refreshed again the messages will be sent again from the cache. Log information will be maintained for this. 


\section{CONCLUSION}

My primary contribution to this paper is to propose a model that involves sufficient connectivity to forward multiple messages to multiple recipients. For forwarding of messages, there should be a reliable message transfer taking place in any technique that is used. Since there are many networks associated with the transferring of a single unit or packet of data, it is difficult to trace the units very easily. Hence gateways (message hubs) are included in MA Server which will interface with JMS clients and help enhance connectivity. Thus by placing the receivers and senders under a single system or object (MA Client), the connectivity and reliable message transfer can be an outcome [1][5][9][12][14].

Enhancing in future can be done by importing the same idea in different applicative areas like computers, web servers, PDA's etc. Since PDA's and computers support multiple emails transfers at a time, this concept if used for easy file sharing through offline approaches like Groove Folder Synchronisation. This enables an offline sharing or transfer of files. There is still a gap that is needed to be fulfilled when it comes to E-Mail (Electronic Mails) where there is no facility for the multiple forwarding of messages. Hence this concept can be used for email forwarding as well.

\section{REFERENCES}

[1] Reliable message delivery for mobile agents: push or pull by Jiannong Cao, Member, IEEE, Xinyu Feng, Jian Lu, Henry C. B. Chan, Member, IEEE, and Sajal K. Das, Member, IEEE.

[2] Gateway:A Message Hub with Store-and-forward Messaging in Mobile Networks by Eiko Yoneki and Jean Bacon University of Cambridge Computer Laboratory, William Gates Building, J J Thomson Avenue Cambridge CB3 0FD, UK \{Eiko.Yoneki, Jean.Bacon\}@ @l.cam.ac.uk

[3] Mobile Agent Communication Mechanism using Sub-Server by Yi-Seok Jeong, Choon-Sung Nam, Hee-Jin Jeong, Dong-Ryeol Shin School of Information and Communication Engineering Sungkyunkwan University 300, ChunChun-Dong, Jangan-Gu, Suwon 440-746, Korea

[4] Tracking mobile units for dependable message delivery by Amy L.Murphy, Gruia-Catalin Roman, George Varghese, IEEE Computer Society.

[5] Method of Forwarding an SMS message in a mobile communication system by Dong-Jun Sin, Seoul (KR).

[6] Message by Message Route Modification in Wireless Multihop Transmission for shorter Delay by Hiroaki Higaki Department of Robotics and Mechatronics Tokyo Denki University Hatoyama Ishizaka, Hiki, Saitama, Japan +81-49-296-2911 Email: hig@ @iglab.net

[7] Large-Scale SMS Messages Mining Based on Map-Reduce by Tian XIA1,2 1 Key Laboratory of Data Engineering and Knowledge Engineering(Renmin University of China), MOE, Beijing 100872, P. R. China 2 School of Information Resource Management, Renmin University of China. Beijing 100872, P.R. China xiat@ruc.edu.cn

[8] Logarithmic Store-Carry-Forward Routing in Mobile Ad Hoc Networks by Jie Wu, Senior Member, IEEE, Shuhui Yang, Student Member, IEEE, and Fei Dai 
[9] Evaluation of Queueing Policies and Forwarding Strategies for Routing in Intermittently Connected Networks (Anders Lindgren, Kaustubh S. Phanse Department of Computer Science and Electrical Engineering Lule'a University of Technology SE-97187 Lulea, Sweden \{dugdale,kphanse\} @ sm.luth.se)

[10] An Adaptive Forwarding Scheme for Message Delivery over Delay Tolerant Networks (Fen Hou and Xuemin (Sherman) Shen University of Waterloo, Waterloo, Ontario, Canada \{fhou, xshen\}@bbcr.uwaterloo.ca)

[11] Optimal Buffer Management Policies for Delay Tolerant Networks (Amir Krifa, Chadi Barakat, Thrasyvoulos Spyropoulos Project-Team Plan`ete, INRIA Sophia-Antipolis, France National School of Computer Sciences (ENSI), Tunisia Swiss Federal Institute of Technology (ETH), Zurich, Switzerland)

[12] Adaptive Multiple-copy Multiple-hop Forwarding In Intermittently Connected Mobile Networks (Jianwei Niu, Hang Zhou BeiHang University Beijing 100083, China, Linmin Sun, Yongping Xiong Institute of Software Academic of Science, Beijing 100080, China)

[13] Peer-Tree: A Peer-to-Peer Message Forwarding Structure for Relaying Messages in Mobile Applications (Jun Liu Computer Science Department, University of North Dakota)

[14] Fragmentation in Store-and-Forward Message Transfer (Barry J. Brachman Samuel T. Chanson)

\section{AUTHORS}

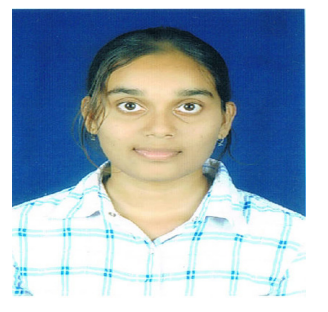

Shivani G.V is currently pursuing his M.S in Software Engineering at VIT University where she focused on Software Engineering methodologies, Computer Networks and Security, and Computer fraud \& Security. Her research interests Includes Mobile Computing and Computer Networks.

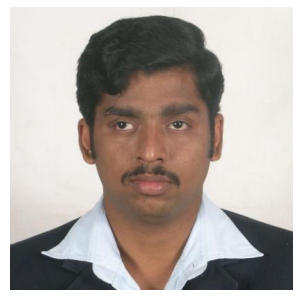

M. Dinakaran has completed his B.Tech (IT) and M.Tech (IT-Networking) in Vellore Institute of Technology, Tamil Nadu, India. He worked in TATA Consultancy Services more than 3 years as Assistant System Engineer. He has awarded as a TCS Gem during third quarter of 2008. Currently he is working as Assistant Professor in VIT University, Vellore and he is pursuing $\mathrm{Ph}$. D in Anna University Coimbatore. He has published 5 articles in International Conferences / Journals. 